\title{
Identifying frailty at the front door
}

\author{
Author: Gary French
}

\section{Aims}

To harness the work already done by the community frailty team in establishing advanced care plans for frail care home residents. Identifying patients with these plans at the front door to ensure clinical teams can be best placed to deliver care which is tailored to patients' own wishes.

\section{Background}

East Sussex Healthcare NHS Trust has a large elderly population. These patients can be complex with several coexisting comorbidities. As a geriatrician I am aware that the acute hospital environment is not always the most appropriate or preferred place of care for many of these patients. There has been an increased move for healthcare providers to provide frailty input at the 'front door' of hospitals.

The trust already has a nurse-led frailty team who visit patients who are highlighted as being frail in the community to undertake a comprehensive geriatric assessment and discuss advanced care planning. It is hoped a more joined-up service which involves the frailty team working in both the community and acute settings will lead to enhanced care for our frail patients.

\section{Methods}

Admissions to the Conquest Hospital under the elderly care take were reviewed over a 4 -week period to identify those patients who had been admitted with an existing advanced care plan in place. Notes were reviewed to see if this care plan had been documented and acted upon. A pilot triage form for emergency department / acute assessment unit will be issued to prompt staff to check and document if an advanced care plan is in place.

\section{Results}

Results are awaited at time of submission.

\section{Conclusion}

We hope to show that early identification of existing advanced care plans can guide the care given to patients by their medical team and aid in patients' wishes being met.

Author: Royal College of Physicians chief registrar, East Sussex Healthcare NHS Trust, UK

\section{Conflict of interest statement}

None. 